\title{
Article
}

\section{The Use of Contrast Therapy in Soft Tissue Injury Management and Post- Exercise Recovery: A Scoping Review}

Greenhalgh, Olivia, Alexander, Jill, Richards, James, Selfe, James and McCarthy, Chris

Available at http://clok.uclan.ac.uk/35883/

Greenhalgh, Olivia, Alexander, Jill ORCID: 0000-0002-6492-1621, Richards, James ORCID: 0000-0002-4004-3115, Selfe, James and McCarthy, Chris (2021) The Use of Contrast Therapy in Soft Tissue Injury Management and PostExercise Recovery: A Scoping Review. Physical Therapy Reviews, 26 (1). pp. 64-72. ISSN 1083-3196

It is advisable to refer to the publisher's version if you intend to cite from the work. http://dx.doi.org/10.1080/10833196.2020.1850163

For more information about UCLan's research in this area go to http://www.uclan.ac.uk/researchgroups/ and search for <name of research Group>.

For information about Research generally at UCLan please go to http://www.uclan.ac.uk/research/

All outputs in CLoK are protected by Intellectual Property Rights law, including Copyright law. Copyright, IPR and Moral Rights for the works on this site are retained by the individual authors and/or other copyright owners. Terms and conditions for use of this material are defined in the policies page. 


\section{The Use of Contrast Therapy in Soft Tissue Injury Management and Post-Exercise Recovery: A Scoping Review}

Olivia Greenhalgh ${ }^{\mathrm{a} *}$, Jill Alexander ${ }^{\mathrm{b}}$, Jim Richards $^{\mathrm{b}}$, James Selfe $^{\mathrm{a}}$ and Chris McCarthy

${ }^{a}$ Department of Health Professions, Manchester Metropolitan University, Manchester, UK;

${ }^{b}$ Allied Health Research unit, Faculty of Health and Wellbeing, University of Central

Lancashire, Preston, UK

*Corresponding author:

Olivia Greenhalgh, PhD Student

Department of Health Professions,

Manchester Metropolitan University,

Manchester, M15 6GX

O.Greenhalgh@mmu.ac.uk

Jill Alexander, Course Lead BSc Sports Therapy

Allied Health Research unit,

Faculty of Allied Health and Wellbeing,

University of Central Lancashire,

Preston, PR1 2HE

JAlexander3@uclan.ac.uk

Jim Richards, Professor of Biomechanics

Allied Health Research unit,

Faculty of Allied Health and Wellbeing,

University of Central Lancashire,

Preston, PR1 2HE

JRichards@uclan.ac.uk

James Selfe, Professor of Physiotherapy

Department of Health Professions,

Manchester Metropolitan University,

Manchester, M15 6GX

J.Selfe@mmu.ac.uk

Chris McCarthy, Clinical Lead of the Manchester Movement Unit,

Department of Health Professions,

Manchester Metropolitan University

Manchester, M15 6GX

Chris.McCarthy@mmu.ac.uk 


\section{The Use of Contrast Therapy in Soft Tissue Injury Management and Post-Exercise Recovery: A Scoping Review}

Background: Contrast therapy is the alternation of thermotherapy and cryotherapy. Commonly used modalities of contrast therapy include contrast water therapy (CWT) and cold/hot packs. Despite a lack of research, it is widely used in clinical and sporting settings, particularly to aid recovery.

Objectives: The scoping review aims to provide a comprehensive overview of research surrounding the use of contrast therapy for soft tissue injury management and recovery.

Major Findings: Twenty-nine full text papers were included, following a search of the databases listed: PubMed, Cochrane, SPORTDiscus, EBSCO, CINHAL and MEDLINE (via OVID). The majority of research on contrast therapy focuses on recovery, using contrast water therapy. Despite a consensus for contrast therapy temperatures of $10-15^{\circ} \mathrm{C}$ (cold) and $38-40^{\circ} \mathrm{C}$ (hot), significant variation amongst recovery protocols still exists, with temperatures ranging from $8-15^{\circ} \mathrm{C}$ and $35.5-45^{\circ} \mathrm{C}$ and duration ranging from 6-31 minutes. Generally, beneficial effects are reported to subjective measures such as self-reported perception of recovery, fatigue and muscle soreness following contrast therapy. However, the evidence is less clear regarding the influence on physiological measures and performance.

Conclusion: Contrast therapy appears to be most commonly used in the form of contrast water therapy for post-exercise recovery purposes. There remains a significant lack of research surrounding the efficacy of contrast therapy for soft tissue injury management and the use of alternative modalities.

Keywords: contrast; cryotherapy; thermotherapy; rehabilitation; recovery

Word Count: 4,534 - including, abstract, tables, references, headings and figure captions 


\section{Introduction}

With athletes often having limited time between training and competition, their ability to recover quickly is becoming increasingly important (1). Contrast therapy is a widely used rehabilitation method for soft tissue injury and post-exercise recovery, despite a lack of research in the field to support its use. Contrast therapy is the alternation of heat (thermotherapy) and cold therapy (cryotherapy) (2). Contrast water therapy (CWT) and cold/hot packs are commonly used modalities of contrast therapy. CWT, alternating hotwater immersion (HWI) and cold-water immersion (CWI), is achieved through full/partial immersion in hot and cold baths/showers in sporting settings to aid recovery. Evidence to support the use of CWT as a recovery strategy has mainly been based upon anecdotal reports (3). Separately, the therapeutic benefits of thermotherapy include an increase in blood flow, metabolism, connective tissue elasticity and pain relief (4). Cryotherapy aims to reduce tissue temperature, perceived pain, cell metabolism, nerve conduction velocity and swelling (5). Despite a general consensus within the literature of the main therapeutic effects of cryotherapy and thermotherapy separately, the physiological basis of contrast therapy is not fully understood.

The purpose of this scoping review is to provide an up-to-date overview of relevant research surrounding the use of contrast therapy for soft tissue injury management and post-exercise recovery.

\section{Methods}

A scoping review was conducted using the Arksey \& O'Malley (6) framework. One author (OG) searched the literature to identify studies relevant to the use of contrast therapy in soft tissue injury management. In order to identify the relevant papers, the following databases were searched: PubMed, Cochrane, SPORTDiscus, EBSCO, 
CINHAL and MEDLINE (via OVID). Hand searches of reference lists were also carried out. The inclusion/exclusion criteria are detailed in Figure 1.
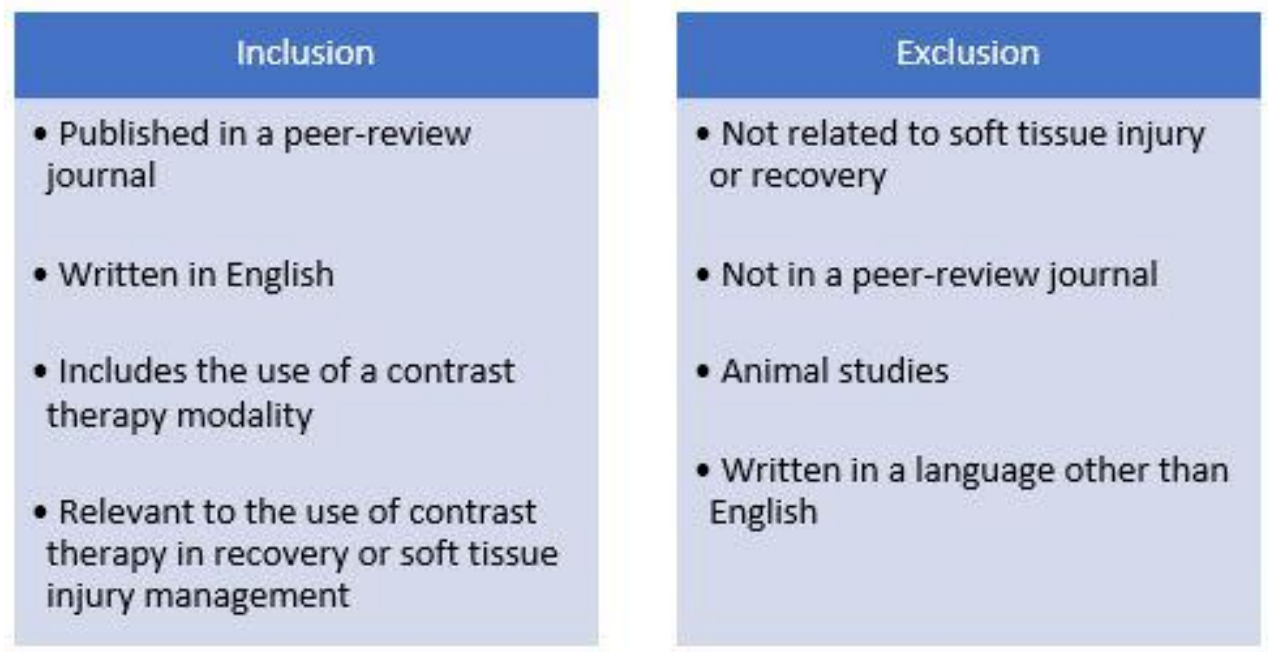

\section{Figure 1: Eligibility Criteria}

The following search terms were used: 'Contrast Therapy' or 'Contrast Water Therapy' or 'Contrast' or 'Thermotherapy' or 'Heat Therapy' or 'Cold Therapy' or 'Cryotherapy'. A wide definition of key words was adopted for search terms as suggested by Arksey \& O'Malley (6) in order to offer a broad coverage of the literature available for the scoping review. Figure 2 illustrates the study selection process in order to identify the relevant sources.

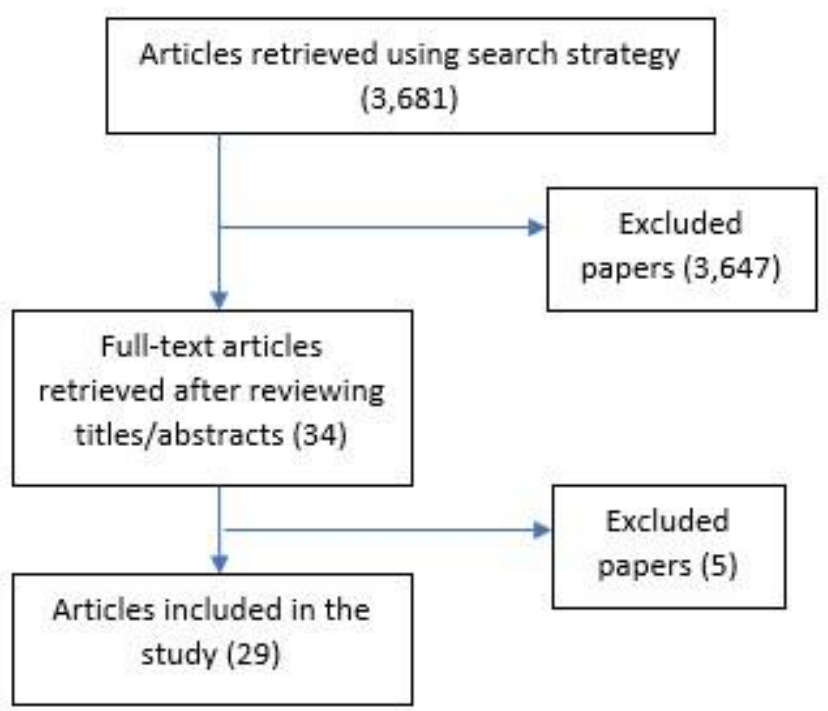

Figure 2: The Study Inclusion/Exclusion Process 


\section{Results}

Twenty-nine full-text papers were included in this scoping review. This consisted of twenty-one research papers, five systematic reviews, with two of those four including a meta-analysis, and three literature reviews. Five papers were excluded following the full-text and abstract review due to duplication or not relating to the use of contrast therapy in soft tissue injury or recovery. Figure 3 provides a breakdown of the characteristics of the studies included in this review.

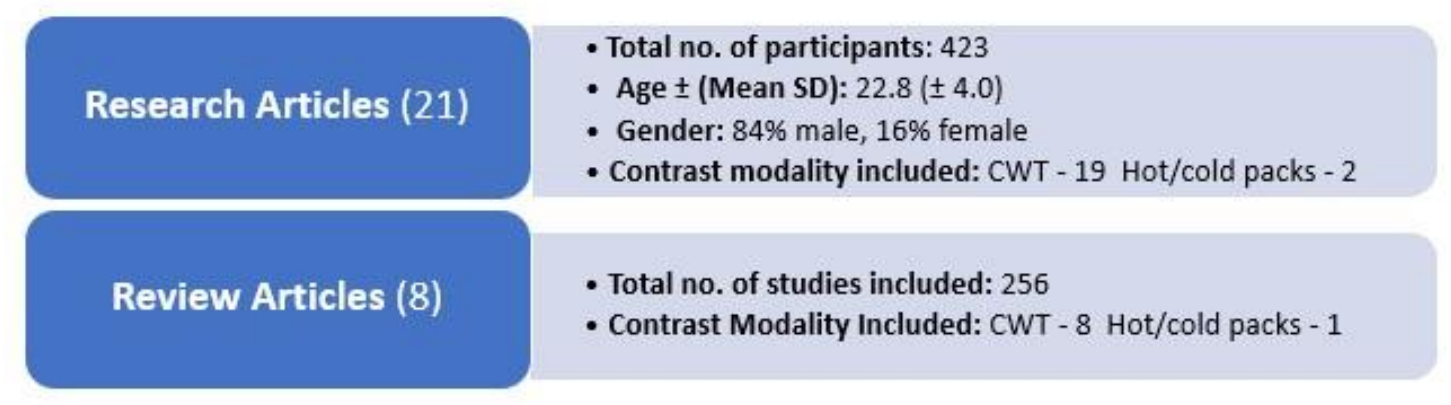

Figure 3: Paper Characteristics Summarised

The majority of the studies included in this review are original research articles. The findings of the review are documented in Table 1. 
Table 1: Data extraction of the included studies

\begin{tabular}{|c|c|c|c|c|c|c|}
\hline \multicolumn{6}{|c|}{ Paper Characteristics } & \multirow[b]{2}{*}{ Key Findings } \\
\hline $\begin{array}{c}\text { Article } \\
\text { Information }\end{array}$ & $\begin{array}{l}\text { Studies } \\
\text { Included }\end{array}$ & Participants & Strategies & Contrast Dose & Purpose & \\
\hline $\begin{array}{r}\text { (Argus et al., } \\
2017) \\
\text { Research } \\
\text { Article }\end{array}$ & & $\begin{array}{l}13 \text { male } \\
\text { volunteers }\end{array}$ & $\begin{array}{l}\text { CWI and } \\
\text { CWT }\end{array}$ & $\begin{array}{l}\text { Full-body } \\
\text { (excluding } \\
\text { head/neck) } \\
38^{\circ} \mathrm{C} 1 \mathrm{~min} / 15^{\circ} \mathrm{C} \\
1 \mathrm{~min}(14 \mathrm{mins} \\
\text { total) }\end{array}$ & Recovery & $\begin{array}{l}\text { - Peak torque during maximal voluntary isometric contraction of the } \\
\text { knee extensors and jump performance were significantly decreased } \\
\text { immediately and } 4 \text { hours post resistance-training in all conditions } \\
\text { - CWI and CWT had no significant effect on performance or } \\
\text { subjective measures during the 4-hour recovery period }\end{array}$ \\
\hline $\begin{array}{r}\text { (Bieuzen et } \\
\text { al., 2013) } \\
\text { Systematic } \\
\text { Review with } \\
\text { Meta-Analysis }\end{array}$ & $\begin{array}{l}18 \mathrm{RCT} \\
\text { or } \\
\text { crossover } \\
\text { trials }\end{array}$ & & $\begin{array}{l}\text { CWT vs } \\
\text { passive } \\
\text { recovery (PR) }\end{array}$ & & Recovery & $\begin{array}{l}\text { Evidence suggests CWT is more effective than PR or rest following } \\
\text { muscle-damaging exercise } \\
\text { - The benefits of CWT include reduced muscle soreness and } \\
\text { increased muscle function (due to a decrease of muscle strength } \\
\text { and power loss) following exercise }\end{array}$ \\
\hline $\begin{array}{r}\text { (Cochrane, } \\
2004) \\
\text { Literary } \\
\text { Review }\end{array}$ & & & CWT & & Recovery & $\begin{array}{l}\text { Previous evidence advocates CWT for reducing injury in the acute } \\
\text { stages, through a shunting action of vasodilation and } \\
\text { vasoconstriction, believed to stimulate blood flow, remove } \\
\text { metabolites, repair muscle and slow down metabolism } \\
\text { - More research is needed to determine whether CWT influences } \\
\text { physiological recovery post-exercise }\end{array}$ \\
\hline $\begin{array}{r}\text { (Crowther et } \\
\text { al., 2017) }\end{array}$ & & $\begin{array}{l}331 \\
\text { athletes }\end{array}$ & $\begin{array}{l}\text { Active } \\
\text { Recovery } \\
\text { (AR) land- } \\
\text { based, AR }\end{array}$ & & Recovery & $\begin{array}{l}\text { - Stretching was considered the most effective recovery technique } \\
\text { - AR land-based was considered the least effective }\end{array}$ \\
\hline
\end{tabular}




\begin{tabular}{|c|c|c|c|c|c|c|}
\hline $\begin{array}{r}\text { Research } \\
\text { Article: Survey }\end{array}$ & & & $\begin{array}{l}\text { water-based, } \\
\text { stretching, } \\
\text { CWI and } \\
\text { CWT }\end{array}$ & & & \\
\hline $\begin{array}{r}\text { (De Nardi et } \\
\text { al., 2011) } \\
\text { Research } \\
\text { Article }\end{array}$ & & $\begin{array}{l}18 \text { teenage } \\
\text { soccer } \\
\text { players }\end{array}$ & $\begin{array}{l}\text { CWI and } \\
\text { CWT }\end{array}$ & $\begin{array}{l}\text { Lower-limb } \\
\text { immersion } 15^{\circ} \mathrm{C} / \\
28^{\circ} \mathrm{C} \text { alternating } \\
\text { every } 2 \text { min }(8 \\
\text { mins total) }\end{array}$ & Recovery & $\begin{array}{l}\text { - CWI and CWT did not influence the athletes' performances } \\
\text { negatively and did not produce changes in inflammatory and } \\
\text { haematological markers in young soccer players } \\
\text { - } \text { Reduced perception of fatigue was the main effect of CWI }\end{array}$ \\
\hline $\begin{array}{r}\text { (Dupuy et al., } \\
\text { 2018) } \\
\text { Systematic } \\
\text { Review with } \\
\text { Meta-Analysis }\end{array}$ & $\begin{array}{l}99 \\
\text { research } \\
\text { articles }\end{array}$ & & $\begin{array}{l}\text { AR, massage, } \\
\text { compression } \\
\text { garments } \\
\text { (GAR), CWI, } \\
\text { CWT, } \\
\text { cryotherapy }\end{array}$ & & Recovery & $\begin{array}{l}\text { - CWT may reduce muscle damage, due to reduced creatine kinase } \\
\text { (CK) concentrations } \\
\text { - CWI and GAR had a positive impact on delayed onset of muscle } \\
\text { soreness (DOMS) and perceived fatigue but not as significantly as } \\
\text { massage }\end{array}$ \\
\hline $\begin{array}{r}\text { (Elias et al., } \\
\text { 2012) } \\
\text { Research } \\
\text { Article }\end{array}$ & & $\begin{array}{l}14 \text { male } \\
\text { Australian } \\
\text { footballers }\end{array}$ & $\begin{array}{l}\text { CWI, CWT, } \\
\text { PR }\end{array}$ & $\begin{array}{l}\text { Full-body } \\
\text { (excluding } \\
\text { head/neck) } \\
\text { Alternating } 38^{\circ} \mathrm{C} \\
1 \mathrm{~min}, 12^{\circ} \mathrm{C} 1 \\
\text { min, }(14 \text { mins } \\
\text { total) }\end{array}$ & Recovery & $\begin{array}{l}\text { - CWI restored physical-performance and psychometric measures } \\
\text { more effectively than CWT } \\
\text { - PR was the least effective }\end{array}$ \\
\hline $\begin{array}{r}\text { (Fiscus et al., } \\
2005) \\
\text { Research } \\
\text { Article }\end{array}$ & & $\begin{array}{l}24 \text { male } \\
\text { volunteers }\end{array}$ & $\begin{array}{l}\text { HWI, CWI, } \\
\text { CWT }\end{array}$ & $\begin{array}{l}\text { Lower-leg } \\
\text { immersion } \\
13^{\circ} \mathrm{C} / 40^{\circ} \mathrm{C}(20 \\
\text { mins total })\end{array}$ & Recovery & $\begin{array}{l}\text { - Contrast therapy produced fluctuations in blood flow } \\
\text { - More research on a larger population should be carried out to } \\
\text { determine the clinical relevance }\end{array}$ \\
\hline $\begin{array}{r}\text { (French et al., } \\
2008)\end{array}$ & & $\begin{array}{l}26 \text { male } \\
\text { volunteers }\end{array}$ & CWT (Baths) & $\begin{array}{l}\text { Lower-limb } \\
\text { immersion }\end{array}$ & Recovery & $\begin{array}{l}\text { - No significant differences in acute recovery from exercise-induced } \\
\text { muscle damage with CWT, compression and PR }\end{array}$ \\
\hline
\end{tabular}




\begin{tabular}{|c|c|c|c|c|c|c|}
\hline $\begin{array}{r}\text { Research } \\
\text { Article }\end{array}$ & & & Compression & $\begin{array}{l}8-10^{\circ} \mathrm{C} 60 \\
\text { seconds } / 37-40^{\circ} \mathrm{C} \\
180 \text { seconds }\end{array}$ & & - CWT may briefly reduce post-exercise soreness \\
\hline $\begin{array}{r}\text { (Gill et al., } \\
\text { 2006) } \\
\text { Research } \\
\text { Article }\end{array}$ & & $\begin{array}{l}23 \text { elite } \\
\text { male rugby } \\
\text { players }\end{array}$ & $\begin{array}{l}\text { CWT, GAR, } \\
\text { low intensity } \\
\text { AR and PR }\end{array}$ & $\begin{array}{l}\text { Lower-limb } \\
\text { immersion } \\
8-10^{\circ} \mathrm{C} \text { for } 1 \\
\min / 40-42^{\circ} \mathrm{C} \text { for } 2 \\
\operatorname{mins}(9 \text { mins } \\
\text { total })\end{array}$ & Recovery & $\begin{array}{l}\text { - Significant increases in CK activity in transdermal exudate found } \\
\text { following rugby } \\
\text { - An enhanced rate and magnitude of recovery was observed with } \\
\text { AR, CWT and GAR at 37-and 84-hours post }\end{array}$ \\
\hline $\begin{array}{r}\text { (Higgins et al., } \\
\text { 2017) } \\
\text { Systematic } \\
\text { Review with } \\
\text { Meta-Analysis }\end{array}$ & 23 articles & & $\begin{array}{l}\text { CWI and } \\
\text { CWT }\end{array}$ & & Recovery & $\begin{array}{l}\text { CWI beneficial for recovery at } 24 \text { hours (countermovement jump) } \\
\text { and } 72 \text { hours (fatigue) post team sport } \\
\text { - CWT beneficial for recovery at } 48 \text { hours (fatigue) and } \\
\text { neuromuscular recovery at } 24 \text { hours post team sport } \\
\text { - Recovery of perceptions of fatigue following team sport were } \\
\text { improved following CWI ( } 72 \text { hours) and CWT ( } 24 \text { hours) } \\
\text { - Neither were beneficial for recovery of perception of muscle } \\
\text { soreness }\end{array}$ \\
\hline $\begin{array}{r}\text { (Higgins et al., } \\
2013) \\
\text { Research } \\
\text { Article }\end{array}$ & & $\begin{array}{l}24 \text { male } \\
\text { rugby } \\
\text { players }\end{array}$ & $\begin{array}{l}\text { CWI and } \\
\text { CWT (shower) }\end{array}$ & $\begin{array}{l}\begin{array}{l}\text { Full-body } \\
\text { immersion }\end{array} \\
10-12^{\circ} \mathrm{C} / 38- \\
40^{\circ} \mathrm{C} \text { for } 60 \\
\text { seconds } \\
\text { alternating (10 } \\
\text { mins total) }\end{array}$ & Recovery & $\begin{array}{l}\text { - No significant difference between heart rate (HR) and blood lactate } \\
\text { - CWT group showed significantly greater DOMS 1-hour post- } \\
\text { intervention than the control group and at 48-hours post- } \\
\text { intervention for the CWI group } \\
\text { - Contrasts baths less effective for recovery than CWI and PR } \\
\text { - Best technique observed for alleviating DOMS following exercise- } \\
\text { induced muscle damage was } 2 \text { x 5-minute CWI }\end{array}$ \\
\hline $\begin{array}{r}\text { (Hing et al., } \\
2008)\end{array}$ & 12 RCT’s & & $\begin{array}{l}\text { CWT } \\
\text { Hot/cold packs }\end{array}$ & & & $\begin{array}{l}\text { The research available regarding the efficacy of contrast therapy in } \\
\text { sports rehabilitation/recovery is lacking quality and quantity } \\
\text { A consensus for temperatures used for contrast therapy (cold: } 10 \text { - } \\
15^{\circ} \mathrm{C} \text { hot: } 38-40^{\circ} \mathrm{C} \text { ) }\end{array}$ \\
\hline
\end{tabular}




\begin{tabular}{|c|c|c|c|c|c|}
\hline $\begin{array}{r}\text { Systematic } \\
\text { Review }\end{array}$ & & & & & \\
\hline $\begin{array}{r}\text { (Juliff et al., } \\
\text { 2014) } \\
\text { Research } \\
\text { Article }\end{array}$ & $\begin{array}{l}10 \text { female } \\
\text { netballers }\end{array}$ & $\begin{array}{l}\text { Passive } \\
\text { Recovery } \\
(\mathrm{PR}), \mathrm{CWT}\end{array}$ & $\begin{array}{l}\text { Full-body } \\
\text { (excluding } \\
\text { head/neck) } \\
\text { Alternating } 1 \mathrm{~min} \\
3^{\circ} \mathrm{C} / 1 \mathrm{~min} 15^{\circ} \mathrm{C} \\
(14 \text { mins total })\end{array}$ & Recovery & $\begin{array}{l}\text { - No significant differences on performance between the recovery } \\
\text { conditions } \\
\text { - Greater heat removal was observed in both water-based recovery } \\
\text { conditions immediately to } 20 \text { minutes post recovery } \\
\text { - Improved perceptions of recovery were observed with the water } \\
\text { recovery conditions in comparison to PR }\end{array}$ \\
\hline $\begin{array}{r}\text { (King \& } \\
\text { Duffield, } \\
\text { 2009) } \\
\text { Research } \\
\text { Article }\end{array}$ & $\begin{array}{l}10 \text { female } \\
\text { netballers }\end{array}$ & $\begin{array}{l}\text { AR, CWI and } \\
\text { CWT }\end{array}$ & 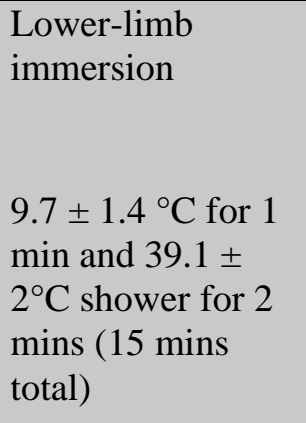 & Recovery & $\begin{array}{l}\text { - No significant differences found in exercise performance during } \\
\text { session } 2 \text { for all conditions } \\
\text { CWT significantly reduced lactate post intervention compared to } \\
\text { AR } \\
\text { - CWT and CWI produced the lowest decline in performance at } \\
\text { session 2, leading to an increased self-reported perceptual recovery }\end{array}$ \\
\hline $\begin{array}{r}\text { (Ménétrier et } \\
\text { al., 2015) } \\
\text { Research } \\
\text { Article }\end{array}$ & 10 athletes & $\begin{array}{l}\text { CWI, CWT } \\
\text { and } \\
\text { thermoneutral } \\
\text { water } \\
\text { immersion } \\
\text { (TWI) }\end{array}$ & $\begin{array}{l}\text { Lower-limb } \\
\text { immersion } \\
12^{\circ} \mathrm{C} 2 \mathrm{mins} / 35^{\circ} \mathrm{C} \\
2 \mathrm{mins}\end{array}$ & Recovery & 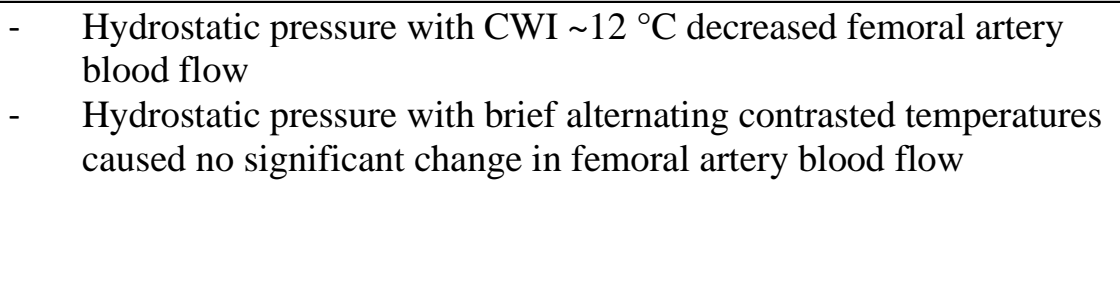 \\
\hline $\begin{array}{r}\text { (Myrer et al., } \\
\text { 1997) } \\
\text { Research } \\
\text { Article }\end{array}$ & $\begin{array}{l}16 \\
\text { volunteers }\end{array}$ & $\begin{array}{l}\text { Hydrocollator } \\
\text { pack } \\
\text { Ice pack }\end{array}$ & $\begin{array}{l}\text { Lower-limb } \\
\text { immersion } \\
\text { Hot-pack/ ice- } \\
\text { pack alternating }\end{array}$ & & $\begin{array}{l}\text { - Contrast therapy has little effect on deep muscle temperature } \\
\text { - } \\
\text { If the physiological effects associated with contrast therapy are } \\
\text { reliant on fluctuations in subcutaneous/intramuscular temperatures, } \\
\text { it would need to be reconsidered as a viable therapeutic modality }\end{array}$ \\
\hline
\end{tabular}




\begin{tabular}{|c|c|c|c|c|c|}
\hline & & & $\begin{array}{l}\text { every } 5 \min (20 \\
\text { mins total })\end{array}$ & & \\
\hline $\begin{array}{r}\text { (Peeling et al., } \\
\text { 2012) } \\
\text { Research } \\
\text { Article }\end{array}$ & $\begin{array}{l}8 \\
\text { endurance } \\
\text { swimmers }\end{array}$ & $\begin{array}{l}\text { CWT } \\
\text { supplemental } \\
\text { oxygen } \\
\text { (HYP), or PR }\end{array}$ & $\begin{array}{l}12^{\circ} \mathrm{C} / 38^{\circ} \mathrm{C} \\
\text { alternating } 1 \mathrm{~min} \\
\text { for } 10 \mathrm{mins} \\
\text { followed by } 10 \\
\text { mins static } \\
\text { stretching, then a } \\
\text { further } 10 \text { mins of } \\
\mathrm{CWT} \\
(20 \text { mins total) }\end{array}$ & Recovery & $\begin{array}{l}\text { - A 30-minute intervention of CWT or HYP has limited effect on the } \\
\text { acute-phase response or on improving performance 1-day post- } \\
\text { intervention } \\
\text { - Acute perception of recovery is improved when an intervention is } \\
\text { implemented compared to no recovery }\end{array}$ \\
\hline $\begin{array}{r}\text { (Pournot et } \\
\text { al., 2011) } \\
\text { Research } \\
\text { Article }\end{array}$ & $\begin{array}{l}41 \text { male } \\
\text { athletes }\end{array}$ & $\begin{array}{l}\text { TWI, CWI, } \\
\text { CWT, PR }\end{array}$ & $\begin{array}{l}\text { Full-body } \\
\text { (excluding } \\
\text { head/neck) } \\
10^{\circ} \mathrm{C} / 42^{\circ} \mathrm{C} 1 \mathrm{~min} \\
30 \mathrm{~s} \text { alternating } \\
\text { (15 mins total) }\end{array}$ & Recovery & $\begin{array}{l}\text { - CWI }\left(10^{\circ} \mathrm{C}\right) \text { and } \mathrm{CWT}\left(10-42^{\circ} \mathrm{C}\right) \text { are the most effective immersion } \\
\text { modalities for maximal anaerobic performance recovery following } \\
\text { exhaustive exercise } \\
\text { - A possible explanation for this could be the removal of plasma } \\
\text { concentrations of markers of inflammation and damage }\end{array}$ \\
\hline $\begin{array}{r}\text { (Stanley et al., } \\
\text { 2012) } \\
\text { Research } \\
\text { Article }\end{array}$ & $\begin{array}{l}18 \text { male } \\
\text { cyclists }\end{array}$ & $\begin{array}{l}\text { CWI, CWT, } \\
\text { PR }\end{array}$ & $\begin{array}{l}\text { Full-body } \\
\text { (excluding } \\
\text { head/neck) } \\
14.2^{\circ} \mathrm{C} 1 \mathrm{~min}, \\
35.5^{\circ} \mathrm{C} 2 \mathrm{mins} \\
\text { alternating (6 } \\
\text { mins total) }\end{array}$ & Recovery & $\begin{array}{l}\text { - CWI most effective at reducing general fatigue, followed by CWT } \\
\text { - CWT most effective at reducing leg soreness, followed by CWI } \\
\text { - Although the effects of CWI and CWT on performance were } \\
\text { insignificant, the improvement on perceptions of recovery provide } \\
\text { a foundation to support the use of these recovery techniques }\end{array}$ \\
\hline
\end{tabular}




\begin{tabular}{|c|c|c|c|c|c|c|}
\hline $\begin{array}{r}\text { (Stanton et al., } \\
2003) \\
\text { Research } \\
\text { Article } \\
\text { (survey) }\end{array}$ & & $\begin{array}{l}43 \\
\text { therapists }\end{array}$ & $\begin{array}{l}\text { CWT } \\
\text { (Contrast } \\
\text { Baths) }\end{array}$ & & Recovery & $\begin{array}{l}\text { - } \quad \text { Contrast baths are commonly used in practice, particularly at home } \\
\text { - } \\
\text { - }\end{array}$ \\
\hline $\begin{array}{r}\text { (Stanton et al., } \\
\text { 2009) } \\
\text { Systematic } \\
\text { Review }\end{array}$ & 10 articles & & CWT & & $\begin{array}{l}\text { Hand } \\
\text { Therapy }\end{array}$ & $\begin{array}{l}\text { - Contrast baths increase superficial blood flow and skin surface } \\
\text { temperature } \\
\text { - }\end{array}$ \\
\hline $\begin{array}{r}\text { (Tavares et al., } \\
2017) \\
\text { Literary } \\
\text { Review }\end{array}$ & & & $\begin{array}{l}\text { CWI and } \\
\text { CWT }\end{array}$ & & Recovery & $\begin{array}{l}\text { In the acute stages ( }<48 \text { hours), the literature suggests that CWI and } \\
\text { CWT can have a beneficial effect on CK clearance, DOMS and } \\
\text { neuromuscular performance }\end{array}$ \\
\hline $\begin{array}{r}\text { (Vaile et al., } \\
2008) \\
\text { Research } \\
\text { Article }\end{array}$ & & $\begin{array}{l}12 \text { male } \\
\text { cyclists }\end{array}$ & $\begin{array}{l}\text { CWI, HWI, } \\
\text { CWT, PR }\end{array}$ & $\begin{array}{l}\text { Full-body } \\
\text { (excluding } \\
\text { head/neck) } \\
\text { Alternating from } \\
15^{\circ} \mathrm{C} \text { for } 1 \mathrm{~min} / \\
38^{\circ} \mathrm{C} \text { for } 1 \mathrm{~min} \\
(14 \text { mins total) }\end{array}$ & Recovery & $\begin{array}{l}\text { CWI and CWT significantly maintained sprint performance greater } \\
\text { than HWI and PR } \\
\text { - CWI and CWT could be effective recovery techniques for events } \\
\text { such as track cycling }\end{array}$ \\
\hline
\end{tabular}




\begin{tabular}{|c|c|c|c|c|c|}
\hline $\begin{array}{r}\text { (Vaile et al., } \\
2008 \text { ) } \\
\text { Research } \\
\text { Article }\end{array}$ & $\begin{array}{l}38 \\
\text { strength- } \\
\text { trained } \\
\text { males }\end{array}$ & $\begin{array}{l}\text { CWI, HWI, } \\
\text { CWT, PR }\end{array}$ & $\begin{array}{l}\text { Full-body } \\
\text { (excluding } \\
\text { head/neck) } \\
\text { Alternating from } \\
15^{\circ} \mathrm{C} \text { for } 1 \mathrm{~min} / \\
38^{\circ} \mathrm{C} \text { for } 1 \mathrm{~min} \\
(14 \text { mins total) }\end{array}$ & Recovery & $\begin{array}{l}\text { - All three hydrotherapy interventions improved isometric force } \\
\text { recovery greater than PR } \\
\text { - CWI and CWT significantly maintained performance greater than } \\
\text { HWI and PR and were more effective in reducing the deficits } \\
\text { associated with DOMS such as: } \\
\text { - Recovery of isometric force and dynamic power } \\
\text { - Reduction of localised swelling } \\
\text { - No significant differences in rate of perceived exertion or HR for any } \\
\text { condition }\end{array}$ \\
\hline $\begin{array}{r}\text { (Versey et al., } \\
2011) \\
\text { Research } \\
\text { Article }\end{array}$ & $\begin{array}{l}11 \text { male } \\
\text { cyclists }\end{array}$ & CWT & $\begin{array}{l}\text { Full-body } \\
\text { (excluding } \\
\text { head/neck) } \\
\text { Alternated } \\
38.4^{\circ} \mathrm{C} / 14.6^{\circ} \mathrm{C} 1 \\
\text { min each }(6,12 \text { or } \\
18 \text { mins) }\end{array}$ & Recovery & $\begin{array}{l}\text { CWT generally improved whole-body fatigue, thermal sensation, } \\
\text { and muscle soreness } \\
\text { - No significant differences between conditions in HR or rating of } \\
\text { perceived exertion } \\
\text { - No apparent dose-response effect on CWT duration on recovery } \\
\text { from high-intensity cycling } \\
\text { - CWT duration up to } 12 \text { mins assisted recovery of cycling } \\
\text { performance }\end{array}$ \\
\hline $\begin{array}{r}\text { (Versey et al., } \\
2012) \\
\text { Research } \\
\text { Article }\end{array}$ & $\begin{array}{l}10 \text { male } \\
\text { runners }\end{array}$ & CWT & $\begin{array}{l}\text { Full-body } \\
\text { (excluding } \\
\text { head/neck) } \\
\text { Alternated } \\
38.4^{\circ} \mathrm{C} / 14.6^{\circ} \mathrm{C} \\
(6,12 \text { or } 18 \text { mins })\end{array}$ & Recovery & $\begin{array}{l}\text { - A 6-minute duration of CWT assisted acute recovery from high- } \\
\text { intensity running } \\
\text { - Algometer pain threshold during CWT for } 12 \text { minutes was higher } \\
\text { compared with the control } \\
\text { - No significant differences in HR or post-exercise calf/thigh } \\
\text { circumferences between conditions } \\
\text { - No apparent dose-response effect on CWT duration and recovery of } \\
\text { running performance } \\
\text { - Subjective measures of thermal sensation and muscle soreness were } \\
\text { lower in all CWT } \\
\text { - No consistent differences following CWT in whole-body fatigue }\end{array}$ \\
\hline
\end{tabular}




\begin{tabular}{|r|l|l|l|l|l|l|}
\hline $\begin{array}{r}\text { (Versey et al., } \\
\text { 2013) } \\
\text { Review Article }\end{array}$ & $\begin{array}{l}53 \\
\text { research } \\
\text { articles }\end{array}$ & $\begin{array}{l}\text { CWI, HWI, } \\
\text { CWT, and } \\
\text { TWI }\end{array}$ & & $\begin{array}{l}\text { Recovery } \\
\text { - It is unlikely that a dose-response relationship between CWT } \\
\text { duration and recovery of exercise performance exists } \\
\text { CWI and CWT more likely to assist recovery of performance than } \\
\text { HWI and TWI }\end{array}$ \\
\hline $\begin{array}{r}\text { (Weerasekara } \\
\text { et al., 2016) } \\
\begin{array}{r}\text { Research } \\
\text { Article }\end{array}\end{array}$ & $\begin{array}{l}118 \\
\text { volunteers } \\
\text { with ankle } \\
\text { sprains }\end{array}$ & $\begin{array}{l}\text { Hot- } \\
\text { fermentation which technique is most effective } \\
\text { heat pack } \\
\text { Ice massage }\end{array}$ & $\begin{array}{l}\text { Lower-leg } \\
\text { immersion } \\
0-10^{\circ} \mathrm{C} / 40-50^{\circ} \mathrm{C} \\
\text { alternating } 1 \mathrm{~min} \\
(15 \text { mins total })\end{array}$ & $\begin{array}{l}\text { Soft } \\
\text { Tissue } \\
\text { Injury }\end{array}$ & $\begin{array}{l}\text { No difference in contrast and heat therapies with ankle ROM } \\
\text { immediately and } 3 \text { days post application } \\
\text { Both modalities increased swelling immediately post application } \\
\text { Contrast therapy reduced swelling } 3 \text { days post but heat therapy } \\
\text { increased swelling after } 3 \text { days } \\
\text { Heat therapy reduced pain immediately post treatment compared to } \\
\text { contrast but no significant difference in reduction of pain after } 3 \text { days } \\
\text { for both modalities }\end{array}$ \\
\hline
\end{tabular}




\section{Discussion}

This scoping review aimed to provide a comprehensive overview of research surrounding the use of contrast therapy for soft tissue injury management and recovery. Twenty-seven (nineteen research articles and eight reviews) out of the twenty-nine studies included in this review used or included CWT as a modality. Only three studies explored alternative contrast modalities which were hot-fermentation and hydrocollator heat packs with ice massage/packs. One review article investigated both CWT and hot/cold packs (7).

Findings from this review suggest that CWT appears to be used predominately as a recovery technique by athletes' post-exercise, with twenty-six out of twenty-seven studies focussing on recovery, opposed to soft tissue injury. However, the evidence surrounding the efficacy of CWT is yet to be fully understood, with the literature reporting conflicting findings on physiological and functional effects. Despite this uncertainty, the perception of recovery is frequently improved with CWT in comparison with a passive recovery technique (8).

In comparison to passive recovery or rest, the reported enhanced therapeutic benefits of CWT include greater heat removal, increased superficial blood flow, fluctuations in blood flow, reductions in creatine kinase (CK) concentrations in the blood, lactate, muscle soreness and an increase in muscle function (9-17). However, studies within this review reported no significant differences in blood lactate, heart rate, femoral artery blood flow or post-exercise calf/thigh circumferences following CWT (18-23). No changes were observed in inflammatory and haematological markers following CWT or CWI (18).

When assessing subjective measures, studies reported that CWT improved selfreported perception of recovery $(24,25)$, perceptions of fatigue following team sport $(8)$ 
and perceived reduction in muscle soreness following exercise (26). Contrastingly, Higgins et al. (8) stated that CWT and CWI were not beneficial for recovery of perception of muscle soreness following team sport. In a survey amongst athletes comparing active recovery, stretching, CWT and CWI, stretching was perceived the most effective recovery technique and active recovery was considered the least effective (27).

The literature presents a mixed response to performance following CWT. Vaile et al. (28) found that cycling sprint performance was improved following 14-minute applications of CWI and CWT compared to HWI and passive recovery. King \& Duffield (24) also reported benefits to performance with CWT and CWI producing the lowest decline in performance compared to passive/active recovery strategies. However, Juliff et al. (9) reported no significant differences on performance between the three recovery conditions (passive, CWT, and contrast showers). Yet, Elias et al. (29) reported that CWT restored physical performance measures more effectively than passive recovery but less effectively than CWI. Overall, the reviewed literature implies a consensus that CWT and CWI are more effective recovery strategies than passive recovery, HWI and thermoneutral-water immersion (TWI). Albeit, despite a number of studies comparing CWT and CWI, it is still unclear as to which immersion technique (CWI or CWT) is most effective.

Versey et al. (30) suggested the optimal CWT conditions for performance recovery would consist of equal immersion between hot/cold baths lasting approximately 1 minute each, for up to 15 minutes. Nevertheless, it is still apparent that there is a lack of standardisation in CWT protocols used for post-exercise recovery (31) and the optimal protocols for other contrast therapy modalities are still unclear. Hing et al. (32) identified a consensus for the temperatures used for contrast therapy for sports 
recovery, across a range of modalities, as $10-15^{\circ} \mathrm{C}$ (cold) and $38-40^{\circ} \mathrm{C}$ (heat). This is in agreement with the defined therapeutic skin surface temperature range of $10-15^{\circ} \mathrm{C}$ used in acute injury management (33). However, Bieuzen et al. (26) noted an inconsistency in the water temperatures being used for CWT; with temperatures ranging from $8-15^{\circ} \mathrm{C}$ (mean: $11.1^{\circ} \mathrm{C}$ ) and $35.5-45^{\circ} \mathrm{C}$ (mean $39.3^{\circ} \mathrm{C}$ ). Immersion techniques also appear to vary; ten studies included in this review used full-body CWT immersion (excluding head/neck), seven immersed the lower-limb and two immersed the lower-leg only Hing et al. (32) also observed durations varied significantly from 6 to 31 minutes amongst the studies using contrast therapy, with ratios alternating from hot to cold ranging from 1:1 to $10: 1$.

Weerasekara et al. (34), the only study in this review surrounding soft tissue injury management, compared the use of contrast and heat therapy using hot fermentation heat pack $\left(40-50^{\circ} \mathrm{C}\right)$ and ice massage $\left(0-10^{\circ} \mathrm{C}\right)$, in subacute grade I and II lateral ankle sprains. Both interventions were applied for 15 minutes; with the contrast alternating hot/cold every minute. The authors advised that contrast therapy is more beneficial for reducing swelling in subacute grade I and II lateral ankle sprains and heat therapy offers greater pain relief initially.

Myrer et al. (2) indicated that contrast therapy using hot/cold packs was unable to produce significant physiological effects on intramuscular temperature $1 \mathrm{~cm}$ below the subcutaneous fat following a 20-minute application. Yet, the authors reported a greater temperature gradient and heat removal, when using ice packs/hydrocollator packs compared to other studies using hydrotherapy.

Hing et al. (7) described the research available on the efficacy of contrast therapy in sports rehabilitation as lacking quality and quantity. Nineteen papers included in this review are subsequent to Hing et al. (32); three of these being 
systematic reviews and eighteen surrounding CWT. The majority of the studies in this review are on small sample sizes, and the majority based on a male population. This highlights that there is still a lack of research providing evidence on the efficacy and therapeutic effects of contrast therapy modalities. This also illustrates that a significant volume of the research on contrast therapy focusses on CWT and post-exercise recovery.

There remains a significant lack of research surrounding the efficacy of contrast therapy in regards to soft tissue injury management and the influence on physiological measures and performance. There is also a significant lack of research using alternative contrast modalities to CWT, which could be due to barriers such as accessibility/availability of other contrast therapy modalities/devices within sporting and clinical settings. The reviewed literature implies a consensus that CWT and CWI are the most effective water immersion recovery strategies. However, further work should aim to standardise an optimal CWT protocol for post-exercise recovery and explore whether contrast therapy could be a viable soft tissue injury treatment. Other contrast modalities should as hot/cold packs and thermal devices should also be considered.

\section{Acknowledgements}

This research was funded by and carried out as part of a Knowledge Transfer Partnership between Manchester Metropolitan University, University of Central Lancashire, and Swellaway Ltd.

\section{Declaration of interest statement}

The authors report no conflicts of interest. 


\section{References}

1. Argus CK, Broatch JR, Petersen AC, Polman R, Bishop DJ, Halson S. ColdWater Immersion and Contrast Water Therapy: No Improvement of Short-Term Recovery After Resistance Training. International journal of sports physiology and performance. 2017;12(7):886.

2. Myrer JW, Measom G, Durrant E, Fellingham GW. Cold and hot-pack contrast therapy: subcutaneous and intramuscular temperature change 1459. Medicine \& science in sports \& exercise. 1997;29(supplement):256. Doi: 10.1097/00005768199705001-01458.

3. Cochrane DJ. Alternating hot and cold water immersion for athlete recovery: a review. Elsevier Ltd; 2004. p. 26-32.

4. Malanga GA, Yan N, Stark J. Mechanisms and efficacy of heat and cold therapies for musculoskeletal injury. Postgraduate medicine. 2015;127(1):57.

5. Bleakley C, McDonough S, MacAuley D. The Use of Ice in the Treatment of Acute Soft-Tissue Injury: A Systematic Review of Randomized Controlled Trials. The American Journal of Sports Medicine. 2004;32(1):251-61. doi: 10.1177/0363546503260757.

6. Arksey H, O'Malley L. Scoping studies: towards a methodological framework. International Journal of Social Research Methodology. 2005;8(1):19-32. doi: 10.1080/1364557032000119616.

7. Hing WA, White SG, Bouaaphone A, Lee P. Contrast therapy - A systematic review. Physcial Therapy in Sport. 2008;9:148-61.

8. Higgins TR, Greene DA, Baker MK. Effects of Cold Water Immersion and Contrast Water Therapy for Recovery From Team Sport: A Systematic Review and Meta-analysis. Journal of Strength and Conditioning Research. 2017;31(5):1443-60. doi: 10.1519/JSC.0000000000001559.

9. Juliff LE, Halson SL, Bonetti DL, Versey NG, Driller MW, Peiffer JJ. Influence of Contrast Shower and Water Immersion on Recovery in Elite Netballers. Journal of Strength and Conditioning Research. 2014;28(8):2353-8. doi: 10.1519/JSC.0000000000000417.

10. Dupuy O, Douzi W, Theurot D, Bosquet L, Dugue B. An Evidence-Based Approach for Choosing Post-exercise Recovery Techniques to Reduce Markers of Muscle Damage, Soreness, Fatigue, and Inflammation: A Systematic Review With Meta-Analysis. Frontiers in Physiology. 2018;9. doi: 10.3389/fphys.2018.00403.

11. Tavares F, Smith TB, Driller M. Fatigue and Recovery in Rugby: A Review. Sports Medicine. 2017;47(8):1515-30. doi: 10.1007/s40279-017-0679-1.

12. Breger Stanton, Lazaro, MacDermid JCBP. A Systematic Review of the Effectiveness of Contrast Baths. Journal of Hand Therapy. 2009;22(1):57-70. doi: 10.1016/j.jht.2008.08.001.

13. Stanley J, Buchheit M, Peake JM. The effect of post-exercise hydrotherapy on subsequent exercise performance and heart rate variability. European Journal of Applied Physiology. 2012;112(3):951-61. doi: 10.1007/s00421-011-2052-7.

14. Pournot H, Bieuzen F, Duffield R, Lepretre P-M, Cozzolino C, Hausswirth C. Short term effects of various water immersions on recovery from exhaustive intermittent exercise. European Journal of Applied Physiology. 2011;111(7):1287-95. doi: 10.1007/s00421-010-1754-6.

15. Gill ND, Beaven CM, Cook C. Effectiveness of post-match recovery strategies in rugby players. British Journal of Sports Medicine. 2006;40:260-3. 
16. French DN, Thompson KG, Garland SW, Barnes CA, Portas MD, Hood PE, et al. The effects of contrast bathing and compression therapy on muscular performance. Medicine and science in sports and exercise. 2008;40(7):1297-306. doi: 10.1249/MSS.0b013e31816b10d5.

17. Fiscus KA, Kaminski TW, Powers ME. Changes in Lower-Leg Blood Flow During Warm-, Cold-, and Contrast-Water Therapy. Archives of Physical Medicine and Rehabilitation. 2005;86(7):1404-10. doi: 10.1016/j.apmr.2004.11.046.

18. De Nardi M, La Torre A, Barassi A, Ricci C, Banfi G. Effects of cold-water immersion and contrast-water therapy after training in young soccer players. The Journal of sports medicine and physical fitness. 2011;51(4):609.

19. Versey NG, Halson SL, Dawson BT. Effect of contrast water therapy duration on recovery of running performance. International journal of sports physiology and performance. 2012;7(2):130.

20. Versey N, Halson S, Dawson B. Effect of contrast water therapy duration on recovery of cycling performance: a dose-response study. Eur J Appl Physiol. 2011;111(1):37-46.

21. Vaile J, Halson S, Gill N, Dawson B. Effect of hydrotherapy on the signs and symptoms of delayed onset muscle soreness. Eur J Appl Physiol. 2008;102(4):447-55.

22. Ménétrier A, Béliard S, Ravier G, Mourot L, Bouhaddi M, Regnard J, et al. Changes in femoral artery blood flow during thermoneutral, cold, and contrastwater therapy. The Journal of sports medicine and physical fitness. 2015;55(78):768.

23. Higgins TR, Cameron ML, Climstein M. Acute response to hydrotherapy after a simulated game of rugby. Journal of Strength and Conditioning Research. 2013;27(10):2851-60.

24. King M, Duffield R. The effects of recovery interventions on consecutive days of intermittent sprint exercise. Journal of Strength and Conditioning Research. 2009;23(6):1795-802.

25. Peeling P, Fulton S, Sim M, White J. Recovery effects of hyperoxic gas inhalation or contrast water immersion on the postexercise cytokine response, perceptual recovery, and next day exercise performance. J Strength Cond Res. 2012;26(4):968-75.

26. Bieuzen F, Bleakley CM, Costello JT. Contrast water therapy and exercise induced muscle damage: a systematic review and meta-analysis. PloS one. 2013;8(4):e62356. doi: 10.1371/journal.pone.0062356.

27. Crowther F, Sealey R, Crowe M, Edwards A, Halson S. Team sport athletes' perceptions and use of recovery strategies: a mixed-methods survey study. BMC sports science, medicine \& rehabilitation. 2017;9(1):6. doi: 10.1186/s13102017-0071-3.

28. Vaile J, Halson S, Gill N, Dawson B. Effect of Hydrotherapy on Recovery from Fatigue. International Journal of Sports Medicine. 2008;29:539-44.

29. Elias GP, Varley MC, Wyckelsma VL, McKenna MJ, Minahan CL, Aughey RJ. Effects of water immersion on posttraining recovery in Australian footballers. International journal of sports physiology and performance. 2012;7(4):357.

30. Versey NG, Halson SL, Dawson BT. Water Immersion Recovery for Athletes: Effect on Exercise Performance and Practical Recommendations. Sports Medicine. 2013;43(11):1101-30. doi: 10.1007/s40279-013-0063-8. 
31. Stanton DB, Bear-Lehman J, Graziano M, Ryan C. Contrast Baths - What do we know about their use? Journal of Hand Therapy. 2003:343-6.

32. Hing WA, White SG, Bouaaphone A, Lee P. Contrast therapy-A systematic review. Physical Therapy in Sport. 2008;9(3):148-61. doi:

10.1016/j.ptsp.2008.06.001.

33. Kennet J, Hardaker N, Hobbs S, Selfe J. Cooling efficiency of 4 common cryotherapeutic agents. Journal of Athletic Training. 2007;42(3):343-8.

34. Weerasekara RMIM, Tennakoon SUB, Suraweera HJ. Contrast Therapy and Heat Therapy in Subacute Stage of Grade I and II Lateral Ankle Sprains. Foot \& ankle specialist. 2016;9(4):307. 